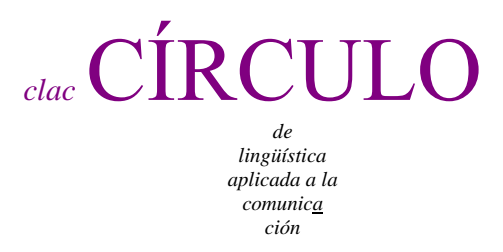

$52 / 2012$

\title{
TITLES IN VETERINARY MEDICINE RESEARCH ARTICLES
}

\author{
Eugenio Cianflone \\ University of Messina \\ ecianflone at unime it
}

\begin{abstract}
Titles are the first point of contact between authors and readers. They call for attention and provide concise and exhaustive information on the research. State of the art in the medical field shows that titles have a mean word count ranging from 15.48 to 15.85 and that they can be arranged into four different formats: nominal, full-sentence, compound and question. Veterinary Medicine has not been object of study and is an underrepresented field in genre analytical surveys. This research wants to fill in part this gap by discussing a pilot survey on veterinary research article titles. To this aim, six issues from three veterinary journals were scanned and the samples labelled "original research article" were examined. The corpus, consisting of 74 specimens, was analysed to elicit the mean word count and the format. Results reveal a mean length of 14.06 words per title and the prevalence of nominal and compound titles. These data are shared to offer a preliminary framework that can serve to inform on the practices adopted by veterinary researchers to communicate findings.
\end{abstract}

Key words: Titles, research article, Veterinary Medicine, Discourse Analysis

Cianflone, Eugenio. 2012.

Titles in Veterinary Medicine research articles.

Círculo de Lingüística Aplicada a la Comunicación 52, 3-20.

http://www.ucm.es/info/circulo/no52/cianflone.pdf

DOI http://dx.org/10.5209/rev_CLAC.2012.v52.41091

(C) 2012 Eugenio Cianflone

Círculo de Lingüística Aplicada a la Comunicación (clac) 52, 3-20.

Universidad Complutense de Madrid. ISSN 1576-4737. http://www.ucm.es/info/circulo 
Contents

1 Introduction 4

2 Materials and Methods 6

2.1 Sample collection 6

2.2 Sample analysis 8

3 Results 9

3.1 The nominal format 10

3.2 The compound format 12

3.3 The full-sentence format 13

3.4 The question format 14

4 Discussion 15

5 Conclusions 16

References 17

1 Introduction

The research article (RA) written in English, even by non-native English speakers, plays a pivotal role in the life of academic communities as it is considered the top medium to validate results before the community of peers (Swales, 2004; Hyland, 2009). In Genre Analysis research, the communicative task of titles has been acknowledged. Titles, in fact, are the first point of contact between authors and readers and play one important function: they stress contents and catch readers' attention when scanning indexes or online search platforms (Haggan, 2004; Gesuato, 2008; Cianflone, 2012). There is an increasing request for guidance on how to encode titles, especially from non-native speakers of English. Analysts in the last decades have met these demands and have produced a consistent number of studies on title encoding practices in different disciplinary fields. Available surveys can be divided into multi-disciplinary and monodisciplinary. 
The former discuss titles in education, psychology and literary criticism (Dillon, 1982); in literature, linguistics and science (Haggan, 2004), in natural, medical and social sciences (Buxton and Meadow, 1977; Jaime Sisò, 2009; Pereira, Fadigas, Senna and Moret, 2011); in anthropology, biology, biochemistry, medicine, linguistics, psychology (Soler, 2007 \& 2011); in medicine, life sciences and physics (Ball, 2009); in medicine and linguistics (Busch-Lauer, 2000); in biology, clinical medicine, oncology, chemistry, engineering and physics (Hartley, 2005 \& 2007; Lewison and Hartley, 2005).

The mono-disciplinary surveys discuss titles in applied mathematics (Yakhontova, 2006); in computer science (Anthony, 2001), in education (Dillon, 1981); in food science (Cianflone, 2012); in linguistics (Gesuato, 2008; Pułaczewska, 2009), in management studies (Forray and Wodilla, 2005); in the humanities (Yitzhaki, 1997) and in psychology (Whissel, 1999). The medical field has also been object of attention. Singh, Chaudhary and Suvirya (2009) examined dermatology samples to study the divergence between the title and the body of the article; Goodman (2000 \& 2005) surveyed the use of active verbs in clinical reports and allusions to Shakespeare's plays, to Andersen's tales, to the Bible, to popular proverbs and films; Siegel, Thacker, Goodman and Gillespie (2006) highlighted the information provided by titles on the topic, the methods, the datasets, the results or the conclusions; Giannoni (2008) discussed titles in medical editorials published in Italian and in English journals; whereas Wang and Bai (2007) examined nominal-group titles.

Data from the surveys discussed above show that in the medical RA the mean length, that is the ratio word per title, ranges from 10.93 words (Wang \& Bai, 2007) to 15.48 (Soler, 2007) with a shift to 15.85 words in recent years (Soler, 2011). The formatting conventions conform to a quadripartite layout, namely nominal structure, compound structure, full-sentence structure and question structure. The prevalence of the first two types (Soler, 2007, 2011) and a general increase in the use of titles with colon (Hartley, 2007) and question mark (Ball, 2009) has been registered.

The impact of the nominal layout mirrors the communicative role played by these titles. Nominal titles, in fact, are selected because they pile-up information by the use of pre- 
modifiers, post-modifiers and, alternatively, verbs. The compound format is the second best choice because the double partition allows authors to exploit the "add-on" principle (Haggan, 2004) to fine-tune the information to be provided. The full-sentence layout allows the discussion of findings with a note of confident optimism in the form of declarative statements (Haggan, 2004; Soler, 2011; Cianflone, 2012), while the question format is considered a means to invite to read on by asking for sympathy or to challenge accepted views (Giannoni, 2008; Soler, 2011).

To date, Veterinary Medicine (VM) titles have not been object of extensive study. The only surveys available are those from Busch-Lauer (2000), Jaime Sisò (2009) and Cianflone (2010). The former two are of the multidisciplinary type and consider VM titles as part of the corpus analysed; whereas the latter describes title formats without quantitative analyses on the frequency of the different layouts in VM practice.

To fill in part this gap, this paper wants to describe the findings, in terms of mean length and of the impact of the different formats, gained from a pilot survey on English RA titles in VM. The research on VM encoding practice was carried out as part of language for specific purposes lectures offered in 2011 to $\mathrm{VM} \mathrm{PhD}$ students of the Faculty of Veterinary Medicine at the University of Messina (Italy).

\section{Materials and Methods}

\subsection{Sample collection}

In literature, corpus compilation has met different criteria. Analysts have selected exemplars labelled "best articles" (Anthony, 1999), the first published articles (Giannoni, 2008), the first 25 specimens from electronic indexes (Gesuato, 2008), or a randomised collection (Anthony, 2001; Forray and Wodilla, 2005; Haggan, 2004; Soler, 2007 \& 2011; Wang and Bai, 2007). As a consequence, analyses were founded on corpora of 30, 40 or 80 specimens per discipline (Giannoni, 2008; Soler, $2007 \& 2011$, 
respectively) or on larger ones when the survey wanted to examine the compound (Hartley, $2005 \& 2007$ ) or the question format (Ball, 2009).

For the educational aims behind the present research, that is to provide a preliminary account of the writing practices used by VM researcher to shape RA titles, the corpus was collected from three international VM journals.

To be selected, journals had to publish research written by native speakers of English and by non-native speakers, so that findings would reflect the writing practices of all VM scholars; journals had to publish papers dealing with different topics to represent the different specialisations peculiar to this disciplinary field; to be included in the survey, papers had also to be labelled "original research article" so that data from analyses would originate from those specimens considered as such by the journals' editorial boards. The selected journals were: The Veterinary Journal (TVJ); the Journal of Equine Veterinary Science (JEVS) and Preventive Veterinary Medicine (PVM). From each journal two issues were randomly selected. The final corpus to be examined, thus, consisted of 74 RA titles.

\begin{tabular}{l|l|}
\hline The Veterinary Journal & vol.184, issue 3=16 RAs. \\
& vol.186, issue 2=14 RAs \\
\hline \multirow{2}{*}{ Journal of Equine Veterinary Science } & Vol.30,issue 4=8 RAs \\
& Vol.30, issue 6=8 RAs \\
\hline Preventive Veterinary Medicine & Vol. 94, issue 3-4 =15 RAs \\
& Vol. 95, issue 3-4 = 13 RAs \\
\hline
\end{tabular}

Research Article Corpus 


\subsection{Sample analysis}

Exemplars were analyzed at the surface level to elicit the mean word count and the frequency of the different structural constructions, namely nominal, compound, fullsentence and question.

In the literature on title analysis, precise instructions on how to count acronyms, numerical sequences and hyphenated words are not given. Because of the presence of these structures within the titles of the examined corpus, and considering that these linguistic constructions are a peculiarity of VM practice, acronyms, numerical sequences and hyphenated words were considered as follows. Acronyms, such as LAMP in title 2, numbers and dates, such as those in title 1, were considered single units and were counted as single words; hyphenated words such as "maternallyacquired", in title 3 , referring to a specific situation or describing a defined condition of the subjects were counted as single words; words indicating animal species were regarded as separate single units and, therefore, considered single words as in title 2 , where in Gadus morhua L, "L" is the shortened form for Linnaeus.

1. Clinical findings and treatment of non-infectious meningoencephalomyelitis in dogs: A systematic review of 457 published cases from 1962 to 2008 (Granger et al., TVJ184(3): 290-297). Total 20 words. 457, 1962 and $2008=$ 3 words

2. Detection of Francisella piscicida in Atlantic cod (Gadus morhua L) by the loop-mediated isothermal amplification (LAMP) reaction (Caipang et al., TVJ, 184(3):357-361). Total 18 words. "L" = 1 word;

3. Dynamics and longevity of maternally-acquired antibodies to Taenia solium in piglets born to naturally infected sows (Sikasunge et 1., TVJ, 184(3): 318321). Total 16 words. Maternally-acquired $=1$ word 


\section{Results}

Titles were manually counted to elicit the mean word count and the frequency of the different layouts. Results show that title length in the analysed corpus ranges from 4 to 24 words. The mean length is attested at 14.06 words per title.

As regards the layout, there is no unanimous consensus on how to label titles. Soler (2007 \& 2011) employs a quadripartite list, namely nominal, compound, full-sentence and question. Haggan (2004) makes use of three tags and defines titles "full sentence", "compound" and "a remaining group". Jaime Sisò (2009) labels titles "indicative" and "conclusive", although the former tag corresponds to the nominal and compound formats, while the latter corresponds to the full-sentence type. Anthony (2001) names compound titles "hanging titles", whereas Hartley (2005 \& 2007) labels them "colonic", thus excluding those titles encoded with the comma, the dash and the full-stop. Soler's (2007 \& 2011) quadripartite account, so far the most comprehensive description, is followed in this survey. Before proceeding further, a description of the different layouts is necessary to introduce the formats that will be discussed in this article.

"Nominal" titles are those arranged around one head, with or without pre-modifiers, followed by other prepositional structures, defined post-modification. The nominal format is divided into non-verbal and verbal (Cianflone, 2012). The latter type is characterised by the presence of one verb that connects the head to the post-modifying structure. Three verbal tenses are used in the nominal verbal format: present participle, past participle and infinitive. Heads, in the form of a noun, a noun-phrase or a nominalized verb in the gerund, introduce the topic, the type of research or the findings, whereas the post-modification contextualises the research space (Cianflone, 2012).

"Compound" titles are those made up of two sections, or interconnected clauses, acting as main and sub-title (Busch-Lauer, 2000). These two parts are mainly linked by a colon, but specimens shaped with a comma, a full-stop or a dash are commonly found in the analysed corpora (see Anthony, 2001). 
"Full-sentence" are those titles phrased as sentences with a subject, referring to the object of study (Jaime Sisò, 2009), followed by a positive or negative declarative verb in the simple present tense, either in the active or in the passive form (Cianflone, 2012) and by an object that fine-tunes the research or the findings discussed in the article (Goodman, 2000).

"Question" are those titles shaped as sentences in the interrogative form (Ball, 2009), that is with the question mark at the very end of the string, where the question is used to raise readers' interest by pointing to the topic by the use of a rhetorical device.

Specimens were, then, analysed to study the frequency and the structural construction. The VM corpus is characterised by the prevalence of the nominal layout, with 56 titles. The compound layout is represented by 14 samples. The full-sentence layout is represented by 3 specimens, followed by only 1 sample in the question format.

\subsection{The nominal format}

Of the 56 nominal titles, 47 are of the non-verbal type, while 9 belong to the verbal type. Both sub-types show structural variety in terms of pre- and post-modification.

As regards the former, in some titles the heads have no pre-modification, as in title 4 or 6 where the head (Carotenodermia and Use, respectively) is directly linked to post-modification. Alternatively, pre-modification is a complex structure made up of several adjectives or of a noun used in adjectival position, as in title 5, where before the head (variation) the adjective single and the noun nucleotide are attached.

Post-modification in VM titles is also characterized by structural variety. In some titles this structure is very simple, as in title 4 , made up of one noun. In other cases it is a complex construction, as in title 2 above or in title 5 below, consisting of a long chain. 
4. Carotenodermia in a Horse (Beech \& Little, JEVS, 30(4): 205-207)

5. Single nucleotide variation in exon 11 of canine BRCA2 in healthy and cancerous mammary tissue (Hsu et al., TVJ, 184 (3): 351-356)

In the verbal nominal titles the heads are connected to the post-modifying structure by a verb. Three types are used in this format: the present participle, the past participle and the infinitive tense.

Of the 9 titles belonging to the nominal verbal format, 4 are in the present participle tense, 3 are encoded with the past participle and 2 with the infinitive tense.

6. Use of a phospholipase-C assay, in vivo pathogenicity assays and PCR in assessing the virulence of Listeria spp (Kaur at al., TVJ, 184(3): 366-370)

7. Factors influencing Salmonella carcass prevalence in Danish pig abattoirs (Baptista et al., PMV, 95(3-4): 231-238)

8. Effect of changes in testing parameters on the cost-effectiveness of two pooled test methods to classify infection status of animals in a herd (Locksley et al., PMV, 94 (3-4): 202-212)

9. A modelling approach to support dynamic decision-making in the control of FMD epidemics (Ge et al., PMV, 95(3-4): 167-174) 
10. Risk factors associated with pleuritis and cranio-ventral pulmonary consolidation in slaughter-aged pigs (Fraile et al., TVJ, 184(3): 326-333)

11. A Bayesian Weibull survival model for time to infection data measured with delay (Kostoulas et al., PMV, 94 (3-4): 191-201)

\subsection{The compound format}

The main feature of the compound format is the partition of the title into two sections, that Busch-Lauer (2000) defines main title and subtitle. This double division does not imply that compound titles are made up of two independent sections. On the contrary, the definition of title and subtitle serves only to indicate that the information is split into two interconnected parts.

The use of different connectors such as the colon, the comma, the dash and the full-stop is reported in literature (e.g. Anthony, 2001). In the examined VM corpus, only one sample, illustrated in title 12, was shaped with the comma, while the remaining 13 titles, are shaped with the colon as illustrated in title 13, 14 and 15.

The interrelationship between the sections follows two different models: "general-specific" and "topic-type of study". In the examined VM corpus the latter prevails, as illustrated by titles 14,15 and 16 .

12. Risk factors for detection of bronchial casts, most frequently seen in endemic H9N2 avian influenza infection, in poultry flocks in Iran (Karimi-Madab et al., PVM, 95 (3-4): 275-280) 
13. Clinical findings and treatment of non-infectious meningoencephalomyelitis in dogs: A systematic review of 457 published cases from 1962 to 2008 (Granger et al., TVJ, 184 (3): 290-297)

14. Prevalence and diagnosis of Babesia and Theileria infections in horses in Italy: A preliminary study (Moretti et al., TVJ, 184 (3): 346-350)

15. Ultrasound-guided block of the sciatic and femoral nerves in dogs: A descriptive study (Echeverry et al., TVJ,186 (2): 210-215)

\subsection{The full-sentence format}

The full-sentence format mirrors the verbal sub-type of the nominal format. It is characterised by the use of a declarative verb either in the present simple or in the present passive tense (Cianflone, 2012). Because of this resemblance to the nominal title, some authors (e.g. Haggan, 2004) include these types within the nominal format. Here, Soler 's descriptions (2007 \& 2011) is followed. Titles shaped with a present tense are, therefore, considered an independent format and not part of the verbal nominal titles because of the specific interactional role they play. The use of the present tense, in fact, characterizes titles in two ways. It gives a note of confident optimism to the message brought out by the title (Soler, 2007) and, at the same time, it allows authors to present the results in an impersonal style that defines an action without disclosing the actor (Hays, 2010). 
Readers' attention in full-sentence titles, in fact, is focussed from the start on the topic at the base of the research or on the findings resulting from the analyses carried out, thus showing a strong advertising imprinting (Berkenrotter \& Huckin, 1995; Jaime Sisò, 2009). The examples quoted below show this interactional value. Title 16 focuses on the effect of tail-biting on the examined animals; title 17 calls attention on blood parameters in the examined cohort of horses.

16. Tail biting induces a strong acute phase response and tail-end inflammation in finishing pigs (Heinonen et al., TVJ, 184 (3): 303-307)

17. Haematological parameters are normal in dominant white FranchesMontagnes horses carrying a KIT mutation (Haase et al., TVJ, 184 (3): 315317)

\subsection{The question format}

The question layout, limited to one specimen, reveals an almost insignificant count with respect to the incidence of the other formats. Similar small counts are in line with the data reported in other surveys (see Soler, 2007 \& 2011).

18. How do features of dressage arenas influence training surface properties which are potentially associated with lameness? (Murray et al., TVJ, 186(2): 172-179) 


\section{Discussion}

The data collected in this survey are not exhaustive because they originate from a small corpus. They offer preliminary insights on the encoding practices employed by VM researchers to conceive a title in terms of mean length and in terms of layout.

The 14.06 figure gained from this survey cannot be compared or contrasted with previous data on VM titles. When compared with data from the medical field, the mean length of VM titles is shorter than the medical counterpart, ranging from 15.48 (Soler, 2007) to a count of 15.85 words in recent years (Soler, 2011). Further surveys are, therefore, necessary to establish whether this difference is the result of the selected corpus or it is a peculiarity of VM writing practices.

One trait in common with the medical RA is the prevalence of the nominal and of the compound format (Soler, 2011). The nominal format, in fact, meets the requests of packaging information in a concise and informative fashion. This interactional peculiarity is obtained in several ways: by the use pre-modifiers, such as adjectives and nouns in adjectival position; by the use of post-modifiers, such as simple nouns or complex structures, together with three different verbal tenses.

The use of verbs to connect heads to the post-modification is a valid alternative to give titles a specific communicative imprint. The present participle and the infinitive tense, as can be inferred from titles 6 to 9 , seem to communicate findings in a fashion that puts authors on a level with readers, thus presenting results in a colloquial fashion. The use of the past participle, on the other hand, seems to inform readers that the whole research is over and that the spreading of findings on a scholarly journal is the last step in the research cycle (Cianflone, 2012).

The consistent presence of compound titles in the examined VM corpus seems to corroborate the growing use of this layout in the medical literature (Hartley, 2007, 2007) and confirms the tendency to use this format as an alternative to the nominal layout (Soler, 2011). The use of the template "topic-type of study" elicited among the VM titles is not in line with data from other disciplinary fields, where the template 
"general-specific" is commonly found among the examined titles (Hartley, 2005; Soler, 2011). It can, therefore, be considered a writing peculiarity of VM titles.

The limited presence of full-sentence titles is not in line with the praxis elicited in other surveys. Elsewhere, the impact of this layout on the writing practices in different disciplines has been connected to the need for a strong communicative imprint that resembles the journalistic style (Berkenrotter \& Huckin, 1995; Haggan, 2004; Jaime Sisò, 2009).

The almost non-existent presence of question titles contradicts the results brought out by other analysts (see Ball, 2009) although it is in line with the writing practices elicited by Jaime Sisò (2009) and Soler (2011). The data gained from this pilot survey may indicate that question titles do not meet VM authors' preferences. Alternatively, the layout can be employed in other written academic genres not considered in the present pilot study, like the editorial or the review paper.

\section{Conclusions}

Titles are classified by genre analysts as independent discourse units (Gesuato, 2008; Haggan, 2004) that stand alone to be catalogued in online indexes or to be retrieved from online search platforms like Google scholar, PubMed or ISI web of science. They play two crucial functions: they catch attention and influence researchers' decision to read on by informing on the topic, on the methods, on the findings and on the impact on everyday practice (Goodman et al., 2001). For this reason, the peculiar traits evidenced by analysts are of two types: informativity and economy (Soler, 2011). The implication is that titles should offer readers as much information as possible with a limited number of words. This is a necessity in contemporary research settings, where 11 million papers can be accessed online (Newmann, 2011) and where the non-informative , or wordy, title can go unnoticed. 
Writing titles, therefore, is a complex activity that asks authors to consider editorial policies, community practices and personal preferences to produce short, catchy and informative strings (Cianflone, 2012). For this reason, the data obtained from this pilot analysis should be considered a snapshot of contemporary VM practices. Results also show how VM researchers communicate findings by concise titles of 14 words and by the use of two formats: nominal and compound titles.

Further analyses with larger corpora are necessary to discover the role the different layouts play in VM. Databases should include not only larger corpora with specimens taken from a more comprehensive collection of VM journals but also other written genres such as editorials, case reports or review papers.

\section{References}

Anthony, Laurence. (1999). "Writing research article introductions in software engineering: How accurate is a standard model?" IEEE Transactions of Professional Communication, 42, 38-46.

Anthony, Laurence. (2001). Characteristic features of research article titles in computer science. IEEE Transactions of Professional Communication, 44, 187-194.

Ball, Rafael. (2009). Scholarly communication in transition: The use of question marks in titles of scientific articles in medicine, life sciences and physics 1996-2005. Scientometrics, 79, 667-679.

Berkenrotter, Carol \& Huckin, Thomas (1995). Genre knowledge in disciplinary communication: Cognition/ culture/power. Hillsdale, NJ: Laurence Erlbaum.

Busch-Lauer, Ines A. (2000). "Titles in English and German research papers in medicine and linguistics”. In A. Trosborg (Ed), Analysing professional Genres (pp. 77-94). Amsterdam: John Benjamins. 
Buxton, Andrew B. \& Meadows, Arthur J. (1977). The variation in the information content of titles of research papers with time and discipline. Journal of Documentation, 33, 46-52.

Cianflone, Eugenio. (2012). Formatting Titles in Food Science. Saarbrücken: LAP.

Cianflone, Eugenio. (2010). Scientific titles in Veterinary Medicine research papers. English for Specific Purposes World, 9, 1-8.

Dillon, J. T. (1982). In pursuit of the colon: A century of scholarly progress: 18801980. Journal of Higher Education, 53, 93-99.

Dillon, John T. (1981). The emergence of the colon: An empirical correlate of scholarship, American Psychologist, 36, 879-884.

Forray Jeanie. M. \& Wodilla Jill (2005). Artefacts of Management Academe: A discourse analysis of temporality in journal titles. Time and Society, 14, 323-339.

Gesuato, Sara (2008). Encoding of information in titles: Academic practices across four genres in linguistics. In C. Taylor (Ed.), Ecolingua: The role of e-corpora in translation and language learning (p. 127-157). Trieste: EUT.

Giannoni, Davide Simone (2008). Disciplinary and linguistic identities in the journal editorial genre. In Di Martino Gabriella, Polese Vanda \& Solly Martin (Eds), Identity and Culture in English Domain-Specific Discourse (p. 325-348). Napoli: Edizioni Scientifiche Italiane.

Goodman, Neville W. (2000). Survey of active verbs in the titles of clinical trial reports. British Medical Journal, 320, 914-15.

Goodman, N. W. (2005). From Shakespeare to Star Trek and beyond: a Medline search for literary and other allusions in biomedical titles. British Medical Journal, 331, 1540-1542.

Goodman, Richard. A., Thacker, Stephen. B., \& Siegel, Paul. Z. (2001). What's in a title? A descriptive study of article titles in peer-reviewed medical journals. Science Editor, 24, 74-78. 
Haggan, Madeleine (2004). Research paper titles in literature, linguistics and science. Dimensions of attraction. Journal of Pragmatics, 16, 293-317.

Hays, Judith C. (2010). Eight recommendations for writing titles of scientific manuscripts. Public Health Nursing, 27, 101-103.

Hartley, James (2005). To attract or to inform: What are titles for? Journal of Technical Writing and Communication, 35, 203-213.

Hartley, James (2007). Planning that title: Practices and preferences for titles with colons in academic articles. Library \& Information Science Research, 29, 553-568.

Hyland, Ken (2009). Academic Discourse. London: Continuum.

Jaime Sisò, Mercedes (2009). Titles or headlines? Anticipating conclusions in biomedical research article titles as a persuasive journalistic strategy to attract busy readers. Miscelánea: a journal of English and American studies, 39, 29-54.

Lewison, Grant \& Hartley, James (2005). What's in a title? Number of words and the presence of colons. Scientometrics, 63, 341-356.

Moore, Andrew (2010). What's in a title? A two-step approach to optimisation for man and machine. Bioessays, 32, 183-184.

Newman, Anthony (2011). How to get published, Power Point Presentation, Maastricht University. http://www.elsevier.com/authored_subject_sections/L07/pdfs/WhatIsPublishing2. pdf [Accessed 09/02/12].

Pereira, Hernane; Fadigas, Inacio; Senna, V. \& Moret, Marcelo A. (2011). Semantic networks based on titles of scientific papers. Physica A, 390, 1192-1197.

Pułaczewska, Hanna (2009).'I bet they are going to read it": Reported direct speech in titles of research papers in linguistic pragmatics. Lodz Papers in Pragmatics, 5, 271-291.

Siegel, Paul Z., Thacker, Stephen B, Goodman, Richard A. \& Gillespie, Cathleen (2006). Titles of articles in peer-reviewed journals lack information on study 
design: A structured review of contributions to four leading medical journals, 1995-2001. Science Editor, 29, 183-185.

Singh, Sanjay; Chaudhary, Rahul \& Suvirya, Swastika (2009). Scientific precision of research papers published in three dermatology journals. Journal of the American Academy of Dermatology Online. doi: 10.1016/j.jaad.2008.11.012

Soler, Viviana (2011). Comparative and contrastive observations on scientific titles written in English and Spanish. English for Specific Purposes, 30,124-137.

Soler, Viviana (2007). Writing titles in science: an exploratory study. English for Specific Purposes, 26, 90-102.

Swales, John (2004). Research Genres. Cambridge: Cambridge University Press.

Wang, Yan \& Bai, Yongquan (2007). A corpus-based syntactic study of medical research article titles. System, 35, 388-399.

Yakhontova, Tatyana (2006). Cultural and disciplinary variation in academic discourse: The issue of influencing factors. Journal of English for Academic Purposes, 5, 153-167.

Yitzhaki, Moshe (1997). Variation in informativity of titles of research papers in selected humanities journals: A comparative study. Scientometrics, 38, 219-229.

Received: September 1, 2012

Accepted: December 6, 2012

Revised: December 21, 2012

Published: December 21, 2012 